\title{
Author Correction: A rare genetic variant of BPIFB4 predisposes to high blood pressure via impairment of nitric oxide signaling
}

\author{
Carmine Vecchione ${ }^{1,2}$, Francesco Villa $\mathbb{1}^{3}$, Albino Carrizzo ${ }^{1}$, Chiara Carmela Spinelli ${ }^{3}$, \\ Antonio Damato ${ }^{1}$, Mariateresa Ambrosio ${ }^{1}$, Anna Ferrario ${ }^{4}$, Michele Madonna ${ }^{1}$, \\ Annachiara Uccellatore ${ }^{5}$, Silvia Lupini ${ }^{5}$, Anna Maciag ${ }^{3}$, Larisa Ryskalin $\mathbb{1}^{6}$, Luciano Milanesi $\mathbb{C}^{4}$, \\ Giacomo Frati ${ }^{1,7}$, Sebastiano Sciarretta ${ }^{1,7}$, Riccardo Bellazzi ${ }^{8,9}$, Stefano Genovese ${ }^{10}$, \\ Antonio Ceriello ${ }^{11,12}$, Alberto Auricchio ${ }^{13,14}$, Alberto Malovini ${ }^{8}$ \& Annibale Alessandro Puca ${ }^{2,3}$
}

Correction to: Scientific Reports https://doi.org/10.1038/s41598-017-10341-x, published online 29 August 2017

In the original version of this Article, Antonio Ceriello was incorrectly affiliated with 'Diabetes Endocrine and Metabolic Diseases Unit, IRCCS MultiMedica, 20099, Sesto San, Giovanni (MI), Italy’ The correct affiliations are listed below.

Institut d'Investigacions Biomèdiques August Pi i Sunyer (IDIBAPS) and Centro de Investigación Biomedica en Red de Diabetes y Enfermedades Metabólicas Asociadas (CIBERDEM), Barcelona, Spain

Department of Cardiovascular and Metabolic Diseases, IRCCS MultiMedica, 20099 Sesto San Giovanni (MI), Italy

This error has now been corrected in the PDF and HTML versions of the Article, and in the accompanying Supplementary Information file.

\footnotetext{
${ }^{1}$ IRCCS Neuromed, 86077, Pozzilli, (IS), Italy. ${ }^{2}$ Department of Medicine and Surgery, University of Salerno, Fisciano, 84084, (SA), Italy. ${ }^{3}$ Cardiovascular Research Unit, IRCCS MultiMedica, 20099, Sesto San, Giovanni (MI), Italy. ${ }^{4}$ Institute of Biomedical Technologies, National Research Council, 20090, Segrate, (MI), Italy. ${ }^{5}$ University of Milan, Via Festa del Perdono, 20122, Milan, Italy. ${ }^{6}$ Department of Translational Research and New Technologies in Medicine and Surgery, University of Pisa, Pisa, 56126, Italy. ${ }^{7}$ Department of Medico-Surgical Sciences and Biotechnologies, Sapienza University of Rome, 04100, Latina, Italy. ${ }^{8}$ Laboratory of Informatics and Systems Engineering for Clinical Research, Istituti Clinici Scientifici Maugeri, 27100, Pavia, Italy. ${ }^{9}$ Department of Electrical, Computer and Biomedical Engineering, University of Pavia, Pavia, Italy. ${ }^{10}$ Diabetes Endocrine and Metabolic Diseases Unit, IRCCS MultiMedica, 20099, Sesto San, Giovanni (MI), Italy. ${ }^{11}$ Institut d'Investigacions Biomèdiques August Pi i Sunyer (IDIBAPS) and Centro de Investigación Biomedica en Red de Diabetes y Enfermedades Metabólicas Asociadas (CIBERDEM), Barcelona, Spain. ${ }^{12}$ Department of Cardiovascular and Metabolic Diseases, IRCCS MultiMedica, 20099, Sesto San, Giovanni (MI), Italy. ${ }^{13}$ TIGEM (Telethon Institute of Genetics and Medicine), 80078, Pozzuoli, Italy. ${ }^{14}$ Department of Translational Medicine, "Federico II" University, Napoli, Italy. Correspondence and requests for materials should be addressed to C.V. (email: cvecchione@unisa.it) or A.A.P. (email: apuca@unisa.it)
} 
(i) Open Access This article is licensed under a Creative Commons Attribution 4.0 International License, which permits use, sharing, adaptation, distribution and reproduction in any medium or format, as long as you give appropriate credit to the original author(s) and the source, provide a link to the Creative Commons license, and indicate if changes were made. The images or other third party material in this article are included in the article's Creative Commons license, unless indicated otherwise in a credit line to the material. If material is not included in the article's Creative Commons license and your intended use is not permitted by statutory regulation or exceeds the permitted use, you will need to obtain permission directly from the copyright holder. To view a copy of this license, visit http://creativecommons.org/licenses/by/4.0/.

(C) The Author(s) 2019 